\title{
Kasuistiken
}

Ophthalmologe 2022 $119: 632-635$

https://doi.org/10.1007/s00347-021-01402-8

Eingegangen: 20. März 2021

Überarbeitet: 2. April 2021

Angenommen: 18. April 2021

Online publiziert: 27. Mai 2021

(c) Der/die Autor(en) 2021

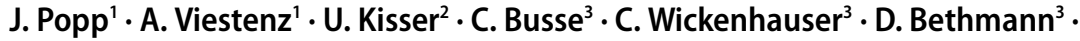
H.-G. Struck' · J. Heichel ${ }^{1}$

'Universitätsklinik und Poliklinik für Augenheilkunde, Martin-Luther-Universität Halle-Wittenberg, Universitätsklinikum Halle (Saale), Halle (Saale), Deutschland

${ }^{2}$ Klinik und Poliklinik für Hals-Nasen-Ohrenheilkunde, Universitätsklinikum Halle (Saale), Halle (Saale), Deutschland

${ }^{3}$ Institut für Pathologie, Universitätsklinikum Halle (Saale), Halle (Saale), Deutschland

\section{Prophylaktische Tränenwegsschienung bei Radiochemotherapie des Saccus lacrimalis}

\section{Anamnese}

Ein 81-jähriger Patient stellte sich mit einer seit 5 Wochen bestehenden rechtsseitigen paranasalen Raumforderung vor (- Abb. 1). Der Mann berichtete über eine intermittierende Epiphora beider Augen (rechts $>$ links). Weitere ophthalmologische Vorerkrankungen bestanden nicht. In der Allgemeinanamnese lagen eine Herzinsuffizienz (NYHA II) und ein rezidivierender Herpes zoster thoracalis vor. Tumorerkrankungen waren nicht bekannt. Eine B-Symptomatik (Fieber, Nachtschweiß, Gewichtsverlust) wurde vom Patienten verneint.

\section{Befund}

Der bestkorrigierte Visus des Patienten betrug rechts 0,4 und links 1,0. Hier war am rechten Auge eine Katarakt vorliegend bei Pseudophakie links. Der Augeninnendruck lag bei $12 \mathrm{~mm} \mathrm{Hg}$ rechts und $9 \mathrm{~mm} \mathrm{Hg}$ links. Sonst fanden sich intraokulär keinerlei Auffälligkeiten.

Im Bereich der rechten Tränensackgrube ließ sich ein derber Tumor tasten (- Abb. 1a, b). Dieser war druckindolent und mit der Unterlage nicht verschieblich (Größe: vertikal 2,5× horizontal 1,8 cm). Es ließ sich kein Sekret aus dem Tränensack exprimieren. Die ableitenden Tränenwege (TNW) waren beidseits über das obere Tränenpünktchen mit leichter
Latenz spülbar. Im konjunktivalen Farbstofftest wurde ein Spontanabfluss von rechts $5 \mathrm{~min}$ und links $3 \mathrm{~min}$ ermittelt.

Die Dünnschicht-Computertomographie (CT) der Orbita und Nasennebenhöhlen zeigte eine verdrängend wachsende Raumforderung des rechtsseitigen Tränensackes (• Abb. 1c). Am Folgetag wurde eine inzisionale transkutane Biopsie in Lokalanästhesie vorgenommen.

\section{Histologische Befunde}

Histopathologisch zeigte sich eine zelldichte Infiltration durch diskohäsiv gelagerte mittelgroße Tumorzellen mit hyperchromatischen vergrößerten Zell-
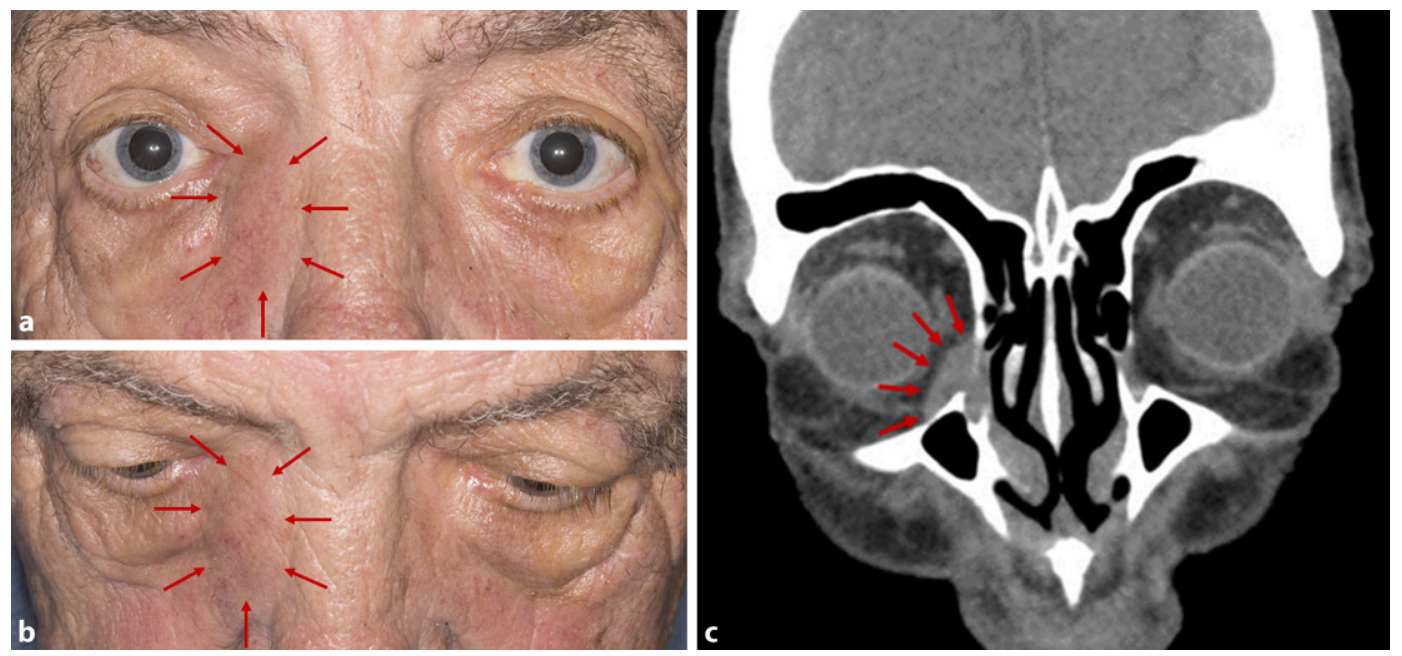

Abb. $1 \varangle$ Klinischer und radiologischer Befund der Raumforderung im Bereich der rechten Tränensackgrube (Pfeile). a Frontale Ansicht. b Aufsicht von oben. c Koronare Schnittebene der Computertomographie (Weichteilfenster) lässt den Tumor des Tränensackes erkennen 

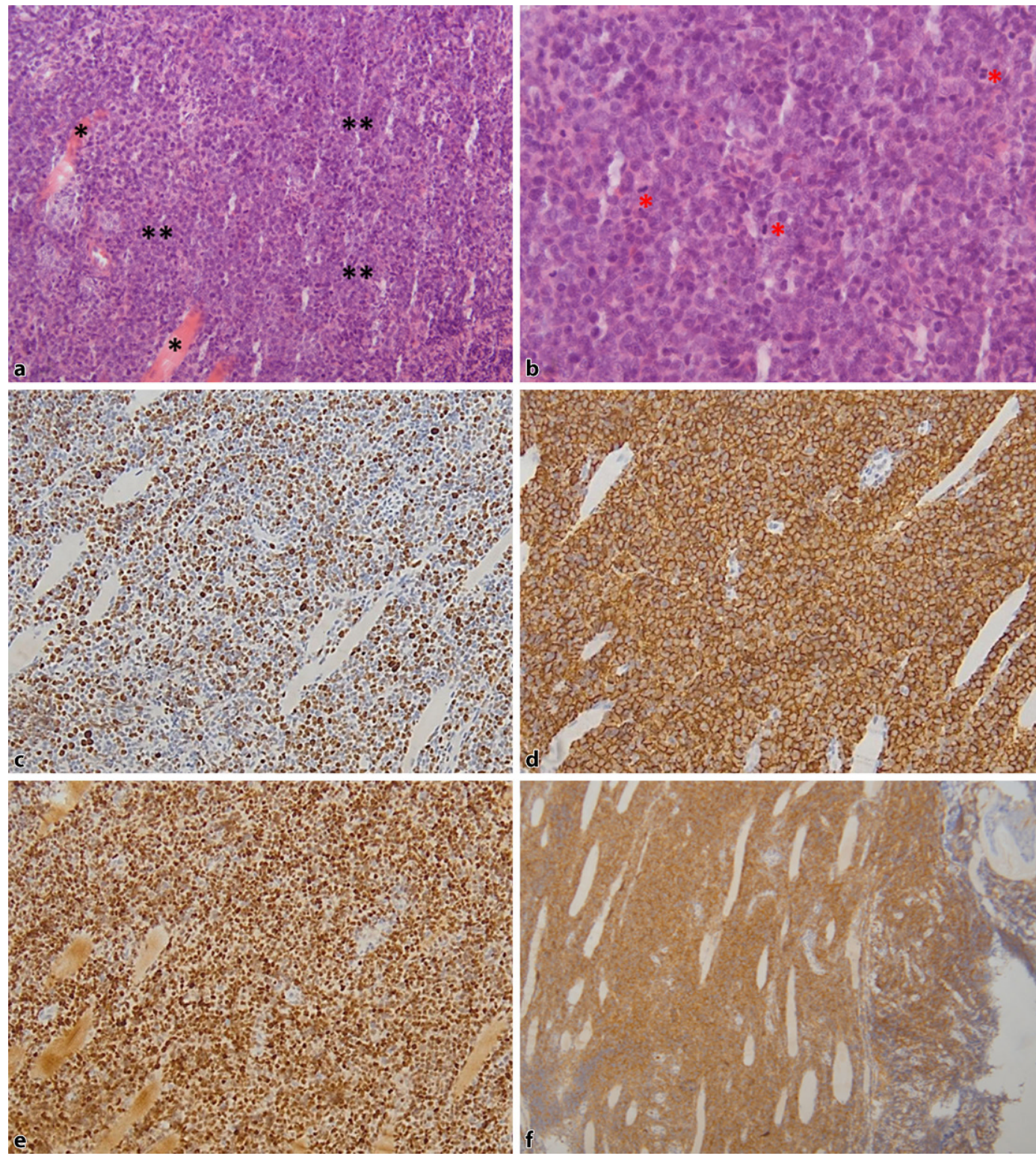

Abb. $2 \varangle$ Histologie und Immunhistologie. a 200fache Vergrößerung, Hämatoxylin-Eosin-Färbung; hochgradige Infiltration ortständiger quergestreifter Muskulatur (Asterisk) durch eine atypische Zellpopulation (2 Asteriske). b 400-fache Vergrößerung, Hämatoxylin-Eosin-Färbung; zelldichte mittelgroßzellige Zellpopulation mit stark erhöhter KernPlasma-Relation und zahlreichen Kernteilungsfiguren (roter Asterisk). c 100fache Vergrößerung, Ki67Färbung (Mib1); nukleäre Färbung in bis zu $70 \%$ der Tumorzellen als Ausdruck einer stark gesteigerten Proliferationsrate. d 100-fache Vergrößerung, immunhistochemische Färbung von CD20; starke membranöse Reaktion in allen Tumorzellen als B-Zell-Marker. e 100-fache Vergrößerung, Immunhistochemie auf bcl 6 („,b cell lymphoma protein 6"); starke nukleäre Färbung in allen Tumorzellen als Surrogatmarker für eine Mutation im BCL6-Gen mit dem Resultat der Apoptoseinhibition. f 100-fache Vergrößerung, immunhistochemische Färbung von CD10; moderate membranöse Färbung in allen Tumorzellen kernen mit Ausbildung von Nukleolen und einer diffusen Infiltration der mitgefassten Muskulatur (• Abb. 2a, b). Die immunhistochemische Ki67-Färbung bestätigte eine stark erhöhte Proliferationsrate bis fokal $70 \%$ ( Abb. 2c). Des Weiteren waren die Tumorzellen reaktiv gegenüber den B-Zell-Markern CD20 (- Abb. 2d) und CD79a mit Koexpression für CD10 und bcl6 (• Abb. 2e, f), entsprechend einer reifen B-Zell-Neoplasie, in erster Linie einem diffus-großzelligen B-Non-Hodgkin-Lymphom, laut des Hans-Klassifikators einem GCB-Typ („germinal center B type“) zuzuordnen. In der Reaktion für CD3 und CD5 zeigt der Tumorverband locker untermischte reaktive T-Lymphozyten.

\section{Weitere Maßnahmen}

Aufgrund der histologischen Diagnose wurde eine Vorstellung im interdisziplinären Tumorboard und eine hämatoonkologische Mitbetreuung eingeleitet (Staging u. a. durch eine Ganzkörper-CT, kraniale Magnetresonanztomographie, Liquorpunktion und Knochenmarkpunktion). Hier ergab sich keine weitere Lymphommanifestation.

Nebenbefundlich zeigte sich ein unklarer Befund im Bereich des rechten
Nebenhodens, welcher einer weiteren Biopsie zugeführt wurde (s. unten).

\section{Diagnose}

Es konnte ein diffus-großzelliges B-ZellLymphom des Tränensackes mit isoliert extranodaler Lokalisation (Ann Arbor: IAE; Germinal Center B-Cell Type) diagnostiziert werden.

\section{Therapie und Verlauf}

Die Biopsie des rechten Nebenhodens wurde wenige Tage später in Allgemeinanästhesie geplant. Bei bereits vorliegender Diagnose des Tränensacklymphoms 


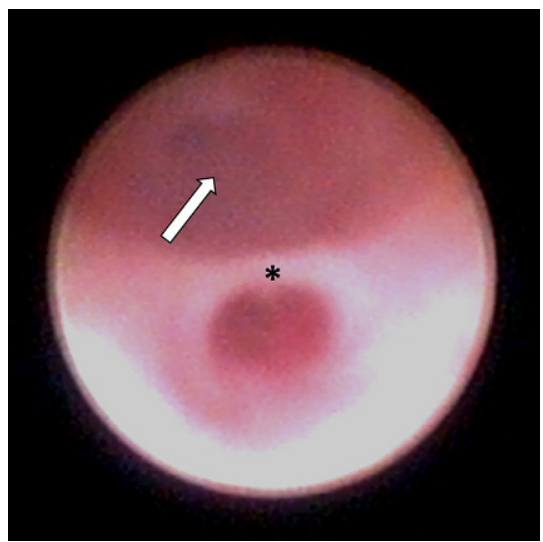

Abb. 3 A Intraoperatives Bild der Dakryoendoskopie. Zu sehen sind eine infundibuläre Einziehung und Stenose am Boden des Tränensackes (Pfeil) sowie ein sich aufspannender Narbenstrang (Asterisk)

wurde in diesem Rahmen eine prophylaktische Schienung der TNW durch 2 autostabile monokanalikulonasale Intubate vorgenommen, da eine Radiotherapie zu erwarten war. Die Histologie des Nebenhodens zeigte eine unspezifische Entzündung und bedurfte keiner weiteren Intervention.

Therapeutisch wurde eine kombinierte Immun- und Radiochemotherapie (IRCT) eingeleitet (R-CHOP-Schema: Rituximab, Cyclophosphamid, Doxorubicin, Vincristin und Prednisolon). Die Bestrahlung wurde als fraktionierte Applikation mittels 18 Zyklen à 2,0 Gy durchgeführt.

Unter der laufenden IRCT kam es zur Luxation mit nachfolgendem Verlust beider TNW-Intubate, da der Patient aufgrund einer Sicca-Symptomatik vermehrt an beiden Augen gerieben hatte. Nach 2 Wochen beklagte er nun eine vermehrte Verschleimung des rechten Auges. Die diagnostische TNW-Spülung zeigte eine absolute mechanische postsakkale Stenose. Dakryoendoskopisch konnte eine weiche, jedoch vollständige Verklebung des Ductus nasolacrimalis bestätigt werden (• Abb. 3). Nach transkanalikulärer dakryoendoskopischer Rekanalisierung wurden erneut 2 autostabile monokanalikulonasale Intubate in die TNW platziert.

Drei bzw. 6 Monate nach Abschluss der IRCT erfolgte die komplikationslose und vollständige Entfernung jeweils eines der Intubate. Die anschließende Tränen- wegsspülung war glatt möglich. Der Patient war zu diesem Zeitpunkt seitens der TNW beschwerdefrei und zeigte einen seitengleichen Spontanabfluss von $3 \mathrm{~min}$. Ein Tumorrezidiv ließ sich klinisch nicht vermuten. In den Magnetresonanztomographien des Kopfes 12 bzw. 18 Monate nach Therapieabschluss zeigte sich ebenfalls kein Anhalt für ein Lokalrezidiv.

\section{Diskussion}

Es ist bekannt, dass Chemotherapien zu zahlreichen okulären Nebenwirkungen führen können und dabei potenziell alle Abschnitte des Auges, von der Adnexe (inklusive Orbita) über das vordere Segment bis hin zum Hinterabschnitt einschließlich den N. opticus, affektieren. Prinzipiell sind alle die Schleimhaut schädigenden Agenzien denkbar, und es ist von einer hohen Dunkelziffer auszugehen $[7,11]$.

TNW-Stenosen infolge von Bestrahlungen sind ebenso vertraut. Jeganathan et al. beschrieben ab einer Strahlendosis von $>65$ Gy Stenosen, Entzündungen sowie dauerhafte narbige Veränderungen der Tränenpünktchen, der Kanalikuli sowie des Tränennasenganges [5]. Aber auch bereits geringere Strahlendosen, wie sie im Rahmen einer Radiojodtherapie durch in der Tränenflüssigkeit gelöstes ${ }^{131} \mathrm{I}$ auftreten, können zu TNWStenosen führen [1].

Das diffuse B-Zell-Lymphom ist weltweit das häufigste der Non-HodgkinLymphome [6]. Es ist typischerweise schnell wachsend und hat seinen Ursprung meist in lymphatischen Organen wie Milz, Lymphknoten oder Knochenmark. Aber auch extralymphatische Gewebe können der Ausgangspunkt sein $[6,10]$. Schmerzlose Lymphknotenschwellung, unklarer Gewichtsverlust, Nachtschweiß und Abgeschlagenheit sind die Leitsymptome dieser Erkrankung [6].

Neben den epithelialen Tumoren sind Lymphome der TNW als häufigste Malignome des Tränensackes zu nennen. Sie werden oftmals durch eine ausgeprägte Epiphora, welche aus einer sekundären Kompression der TNW resultieren, symptomatisch. Weiterhin finden sich indolente Raumforderungen der Tränensackgrube [8, 14]. Als Tumorkomplikation kann es jedoch auch zu kompressionsbedingten Tränenabflussstörungen und somit (sub)akuten Dakryozystitiden kommen, welche mit einem entzündungsbedingten Schmerzreiz einhergehen [15].

Die häufigsten Lymphome des Tränensackes sind das diffuse B-Zell-Lymphom und MALT-Lymphome (extranodale Marginalzonen-B-Zell-Lymphome) $[9,13,14]$. Isolierte und multifokal systemische Formen sind zu unterscheiden. Die Angaben zum Vorkommen dieser gesonderten TNW-Beteiligungen variiert in den Literaturangaben. In den größeren Fallberichten zeichnet sich eine Häufigkeit von etwa 10-20\% ab [4, 8, 9, 13-15].

Die Behandlung eines isolierten TNW-Lymphoms beinhaltet eine Chemotherapie, eine Radiatio oder ggf. eine Kombination aus beidem. Die interdisziplinäre Patientenbetreuung ist dabei unerlässlich $[3,8,10,12,14]$. Eine prophylaktische Schienung wird sowohl bei Chemotherapie als auch Radiatio im Bereich der TNW empfohlen [2, $5,7,15]$. De Smet et al. untersuchten das Auftreten einer sekundären TNWStenose nach Radiotherapie. Sie beobachteten eine postradiogene Epiphora bei $31 \%$ ihrer Patienten ohne absichernde TNW-Schienung, wohingegen dieser Wert auf $6 \%$ infolge einer prophylaktischen TNW-Intubation gesenkt werden konnte [2]. Für die Strahlentherapie eines Lymphoms des Tränensackes werden kumulative Dosen von 30-45Gy empfohlen $[3,12]$.

Der Fall eines extranodalen B-ZellLymphoms im Bereich des Tränensackes wurde hier dargestellt. Im Rahmen der IRCT kam es nach Verlust der prophylaktischen TNW-Schienung zu einer sekundären TNW-Stenose. Da die systemische Chemotherapie an beiden TNW Einfluss genommen hätte, ist von einer vornehmlich postradiogenen Stenose auszugehen, wenngleich eine zusätzliche Einwirkung durch die Chemotherapie nicht ausgeschlossen werden kann. Auch ein chirurgisches Trauma als Ursache der Stenose ist denkbar. Durch eine wiederholte TNW-Intervention mit Rekanalisierung und erneuter Schienung konnte eine po- 
tenziell finale Vernarbung der TNW vermieden werden.

\section{Fazit für die Praxis}

\section{- Eine Stenose der Tränennasenwege kann als Komplikation nach oder während einer Chemotherapie oder Radiatio im Gesichtsbereich auftre- ten. \\ - Die prophylaktische Schienung der Tränennasenwege bietet eine Mög- lichkeit zur Vermeidung postthera- peutischer Stenosen. Dabei sollte der gesamte ableitende Tränenapparat durch entsprechende Silikonverweil- sonden gesichert werden.}

\section{Korrespondenzadresse}

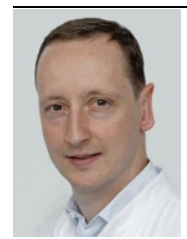

PD Dr. med. habil. J. Heiche

Universitätsklinik und

Poliklinik für Augenheilkunde, Martin-Luther-Universität Halle-Wittenberg, Universitätsklinikum Halle (Saale)

Ernst-Grube-Str. 40, 06120 Halle (Saale),

Deutschland jens.heichel@uk-halle.de

Funding. Open Access funding enabled and organized by Projekt DEAL.

\section{Einhaltung ethischer Richtlinien}

Interessenkonflikt. J. Popp, A. Viestenz, U. Kisser C. Busse, C. Wickenhauser, D. Bethmann, H.-G. Struck und J. Heichel geben an, dass kein Interessenkonflikt besteht.

Für diesen Beitrag wurden von den Autoren keine Studien an Menschen oder Tieren durchgeführt. Für die aufgeführten Studien gelten die jeweils dort angegebenen ethischen Richtlinien. Für Bildmaterial oder anderweitige Angaben innerhalb des Manuskripts, über die Patienten zu identifizieren sind, liegt von ihnen und/oder ihren gesetzlichen Vertretern eine schriftliche Einwilligung vor.

Open Access. Dieser Artikel wird unter der Creative Commons Namensnennung 4.0 International Lizenz veröffentlicht, welche die Nutzung, Vervielfältigung, Bearbeitung, Verbreitung und Wiedergabe in jeglichem Medium und Format erlaubt, sofern Sie den/die ursprünglichen Autor(en) und die Quelle ordnungsgemäß nennen, einen Link zur Creative Commons Lizenz beifügen und angeben, ob Änderungen vorgenommen wurden.
Die in diesem Artikel enthaltenen Bilder und sonstiges Drittmaterial unterliegen ebenfalls der genannten Creative Commons Lizenz, sofern sich aus der Abbildungslegende nichts anderes ergibt. Sofern das betreffende Material nicht unter der genannten Creative Commons Lizenz steht und die betreffende Handlung nicht nach gesetzlichen Vorschriften erlaubt ist, ist für die oben aufgeführten Weiterverwendungen des Materials die Einwilligung des jeweiligen Rechteinhabers einzuholen.

Weitere Details zur Lizenz entnehmen Sie bitte der Lizenzinformation auf http://creativecommons.org/ licenses/by/4.0/deed.de.

\section{Literatur}

1. Burns JA, Morgenstern KE, Cahill KV, Foster JA, Jhiang SM, Kloos RT (2004) Nasolacrimal obstruction secondary to I(131) therapy. Ophthalmic Plast Reconstr Surg 20:126-129

2. de Smet MD, Buffam FV, Fairey RN, Voss NJ (1990) Prevention of radiation-induced stenosis of the nasolacrimal duct. Can J Ophthalmol 25:145-147

3. Esik O, Ikeda H, Mukai K, Kaneko A (1996) A retrospective analysis of different modalities for treatment of primary orbital non-Hodgkin's lymphomas. Radiother Oncol 38:13-18

4. Flanagan JC, Stokes DP (1978) Lacrimal sac tumors. Ophthalmology 85:1282-1287

5. Jeganathan VS, Wirth A, MacManus MP (2011) Ocular risks from orbital and periorbital radiation therapy: a critical review. Int J Radiat Oncol Biol Phys 79:650-659

6. Li S, Young KH, Medeiros LJ (2018) Diffuse large B-cell lymphoma. Pathology 50:74-87

7. Mansur C, Pfeiffer ML, Esmaeli B (2017) Evaluation and management of chemotherapy-induced epiphora, punctal and canalicular stenosis, and nasolacrimal duct obstruction. Ophthalmic Plast Reconstr Surg 33:9-12

8. Montalban A, Liétin B, Louvrier C, Russier M, Kemeny JL, Mom T, Gilain L (2010) Malignant lacrimal sac tumors. Eur Ann Otorhinolaryngol Head Neck Dis 127:165-172

9. Pe'er JJ, Stefanyszyn M, Hidayat AA (1994) Nonepithelial tumors of the lacrimal sac. Am J Ophthalmol 118:650-658

10. Pfreundschuh M (2016) Aktuelle Therapiestrategien beim diffusen großzelligen B-Zell-Lymphom Internist 57:214-221

11. Schmid KE, Kornek GV, Scheithauer W, Binder S (2006) Update on ocular complications of systemic cancer chemotherapy. Surv Ophthalmol 51:19-40

12. Smitt MC, Donaldson SS (1993) Radiotherapy is successful treatment for orbital lymphoma. Int J Radiat Oncol Biol Phys 26:59-66

13. Stefanyszyn MA, Hidayat AA, Pe'er JJ, Flanagan JC (1994) Lacrimal sac tumors. Ophthal Plast Reconstr Surg 10:169-184

14. von Goscinski C, Koch KR, Cursiefen C, Heindl LM (2016) Tumoren der ableitenden Tränenwege. HNO 64:386-393

15. Yip CC, Bartley GB, Habermann TM, Garrity JA (2002) Involvement of the lacrimal drainage system by leukemia or lymphoma. Ophthal Plast Reconstr Surg 18:242-246

\section{Autoren WERKSTATT}

GRATIS

Ein Service für Autoren von Springer Medizin

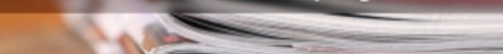

Fortbildungen für

Autor*innen und Gutachter*innen

Die ersten Veröffentlichungen sind für Jeden ein wichtiger Schritt in der angestrebten Karriere. Wissenschaftliche Artikel sind entscheidend dafür, dass die eigene Arbeit in der Community wahrgenommen wird. Es geht darum, die eigenen Ideen national und international auszutauschen und sicherzustellen, dass die Ergebnisse Wirkung erzielen.

Die Online-Kurse der Autorenwerkstatt helfen, sich leicht einen Überblick über das Schreiben, Einreichen, Begutachten und Veröffentlichen eines Manuskripts zu verschaffen.

5 Online-Kurse zu den wichtigsten Standards des wissenschaftlichen Publizierens:

- Wie verfasse ich ein Manuskript?

- Writing in English für deutschsprachige Autorinnen und Autoren

- Wie funktionieren Publikation und Begutachtung?

- Anleitung zur Open-AccessVeröffentlichung

- Leitfaden zur Peer-ReviewBegutachtung

Für alle, die auf SpringerMedizin.de registriert sind!

Jetzt gratis fortbilden unter www.springermedizin.de/ autorenwerkstatt/ 